


\title{
Experiences in Authoring Hypermedia: Creating Better Presentations
}

\author{
Lynda Hardman \\ CWI, Amsterdam, The Netherlands
}

\section{Introduction}

The first part of this chapter discusses the lessons that have been learned by the author during her involvement with a number of academic and commercial hypertext projects. This experience has been gained through conducting controlled reader studies, where inadequacies of the final document interface can be found, and through observations of authors trying to create material in the new medium and not being aware of sources of applicable design knowledge. This part concentrates on what needs to be considered during the different stages of design and assumes that the author is following some form of structured design process, such as that described in (Garzotto et al. 1994). The second part motivates and describes a structured approach to authoring hypermedia. While the editing environment described does not explicitly support the requirements discussed in the first part, the author can more easily experiment with and change both the structure and layout of the presentation until s/he is satisfied. A final section looks to further possible improvements in simplifying hypermedia authoring.

\section{Designing for the Reader}

Although creating hypermedia presentations is a complex task there exists little advice about what factors authors need to take into consideration when designing and creating such presentations. Authoring hypermedia information can be compared with other creative processes, such as writing a book, structured programming, designing an exhibition or making a film. In all these cases authors need to be clear about the ideas they want to get across, how these will be structured, the best media for expressing each idea, how these will be layed out, and navigation routes through the material. Before embarking on the creation process, authors need to consider all of these diverse design aspects.

Three main issues of hypermedia design are addressed in this section:

- How should information be structured?

- How should the information be presented?

- What tools do readers need for exploring the information? 


\subsection{Information structure}

An obvious advantage of using hypermedia is that it can reflect the "natural" structure of the subject matter being dealt with. For example, the medical domain often uses distinct functional and anatomical structures for classifying information, and one, or indeed both, of these structures can be reflected in the hypermedia structure. This advantage is more apparent in complex domains where there are multiple ways of structuring the material. It was for this reason that the creator of the neuroanatomy tutorial in (Hardman 1988) chose to use hypertext as the communication medium. She found it difficult to explain all the different related aspects of neuroanatomy to her students and sought a way of more directly communicating the different approaches and their connections.

Given that this is what the author wishes to achieve, how does an author know when hypermedia information is "well-structured"? At a high level this can be expressed as giving a coherent reflection of the subject matter, that there are sufficient options for navigation and that the structure supports the task the user is carrying out. While this does not take us much further towards concrete design rules, we can learn from analysing what has worked, or not, in previous designs, as discussed in this section.

\section{Effective structure}

Many systems enable the creation of hypertext structure but give an author no support in determining what would be the most effective structures to create. Creating successful structure is often still a matter of seeing what works, but a small number of studies have been carried out to look at the positive and negative effects of different approaches to structuring in hypertext. For example, the study described in (Edwards \& Hardman 1989) was devised to look at how hypertext structures can help or hinder readers in accessing information. Different groups were exposed to the same information structured in three different ways: a hierarchy access structure, an index structure and a dual access structure (index plus hierarchy). The hope was that the dual access structure would be the preferred form of structuring, because it offered more ways of accessing the information. In fact this was perceived by the readers to be the least helpful of the structures, probably because they had too little time with the material to be able to adjust to the extra complexity.

This experiment warns against the use of too many links which, while bringing the advantages of more flexible access structures, have to be weighed against the cost of the extra complexity. This finding is confirmed by Wright (1991), who demonstrated that "one click away" is not always as close for the reader as the designer may think - for inexperienced computer users accessing information via a link involves a cognitive load, thus disturbing readers in their train of thought.

An example of successful consistent structuring of information is the Glasgow Online hypertext (Hardman 1989). The hypertext has several different sections with information about the city of Glasgow, and by using the same types of 
structuring throughout the hypertext readers could easily use sections they hadn't previously visited based on their experience with sections they had.

\section{Content-based vs. navigational structures}

When designing hyper-structure it is useful to distinguish between different types of structure - in particular content-based and navigational structures. A similar distinction is made by (Hannemann \& Thuring 1994) as factual and rhetorical structuring, and by (Garzotto et al. 1994) as hyperbase schema design, reading schema(s) design, instance schemas design and authoring in the small, versus access structure(s) design. The content-based and navigational-based structures are illustrated by the hierarchy and index access structures, respectively, in the Edwards \& Hardman experiment. The hierarchical structuring is a content-based structure since it gathers parts of the material into meaningful groups. The index is a navigational structure in that it gives the reader access to the information but implies no further relation between the source and destination of the link. The design process should start by considering the content-based structures, since these reflect the material the reader is interested in, and then adding the navigational structures "on top" of these.

\section{Application of HCI principles}

Just as in the building of any computer application, a hypermedia author can be helped by considering human computer interaction principles and guidelines. (Hardman \& Sharratt 1989) give a distillation of these which have been specialised to hypertext design ${ }^{\dagger}$. Examples of design principles applied to hypertext are (paraphrased):

Mental processing The hypertext should not complicate the reader's information gathering tasks nor impose excessive mental processing requirements. The author should: (i) minimize the overall mental load by reducing the requirement for readers to remember objects, actions, codes and abbreviations; (ii) minimize the task-specific mental processing by arranging for efficient completion of a typical reader task.

Flexibility The hypertext should: (i) be capable of adaptation to the needs of readers of different types and levels of experience; (ii) provide multiple paths that allow readers to by-pass parts of the hypertext.

A selection of these design principles have been applied in the creation of a hypermedia document describing a tour of Amsterdam (Figure 1): the amount of information presented is kept to a minimum; different routes are provided (the reader is always able to return to the initial screen, can choose to go back to the beginning of the walking tour, or can go on to the next stop on the tour).

$\dagger$. The principles and guidelines discussed in the paper need to be extended to hypermedia presentations which also include dynamic media such as sound and video. 
Although the design can be improved by the application of these principles and guidelines, the most influential factors on hypertext usability are individual differences among users, so that, disregarding how much effort went into "good" design, the system still needs to be evaluated with representative users (Nielsen 1989).

\subsection{Information presentation}

However good the structural design of a hypertext document is, it still needs to be expressed using good presentation. There are two separate aspects of information presentation which need to be addressed. First, exactly what information needs to be present on the screen, for example, sufficient contextual information at the destination of a link for readers to be able to orient themselves. Second, the (typo)graphic design of this information, for example, titles should be placed at the top of the window in a large, bold font.

When deciding on what and how much information should be presented, issues such as screen clutter have to be weighed against the cognitive overhead of not having the information visible. (Hardman \& Sharratt 1989) contains a relevant selection of guidelines (distilled from existing human computer interaction guidelines), for example, in paraphrased form:

- Necessary information, for example, the name of the current section, a title, the information of interest, and the link markers should be displayed.

- Different link types, for example those leading to a small note or those that jump to an entirely new section, should be apparent (before the reader is committed to following the link).

Having decided upon what information is to be presented this has to be translated into some visual form that allows the reader to interact with it. For example, that a link can be followed has to be apparent to the reader. This requires either some form of visual encoding for the link-ends, or informing the reader which objects are likely to form the ends of links. When a visual encoding is taken from existing typographic conventions readers have problems recognising

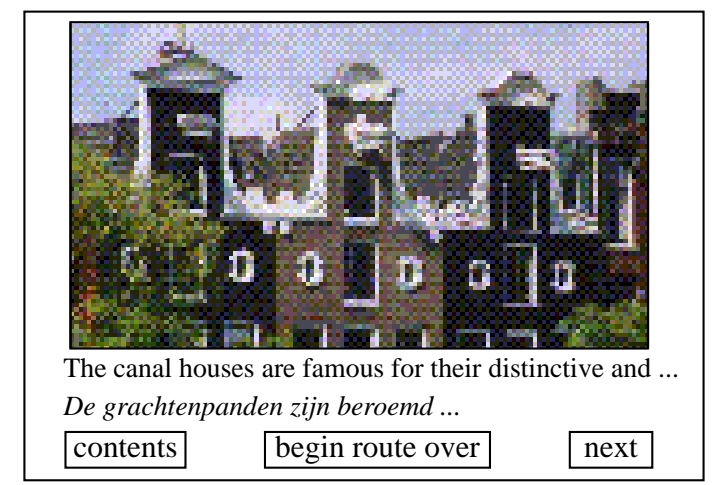

Figure 1. The gables clip from a walking tour in Amsterdam. 
the link-ends. Advantages of this approach, however, are that no new symbols or markup are required and that the screen remains relatively clutter-free. Most systems introduce new symbols or box items to denote link-ends (where some are more aesthetically pleasing than others). Creative approaches to designing link markers are described in (Evenson \& Rheinfrank 1989) and (Kahn 1994).

Some consequences of confronting readers with less than optimal presentation conventions are described in (Hardman 1989). For example, linked items and non-linked items were often confused because inconsistent typographic styles were used. Some conclusions about presentation issues from this study are the following:

- Conventions can be used to convey information and reduce screen clutter.

- Conventions need to be explained to the reader and used consistently.

- Contextual information can be passive, or active, allowing readers to use it to move to different sections (for example, a list of section headings indicating the current section could be used to jump to the other sections).

- Readers should be able to tell which screen items are link-markers.

- Inactive items that require emphasis should not look like link-markers.

- Different styles of link-markers (indicating different link types) can be used at the cost of added complexity.

For information about on-screen design in general (Kahn \& Lenk 1993) pulls together a wealth of experience in the area.

\subsection{Navigation tools}

In the online environment there is no physical presence of stored information; it is difficult to give an overview about how much information there is, or "where" some pieces of information lie with respect to each other. To overcome some of the shortcomings of the electronic environment the reader needs to be provided with, for example, a suite of (simple to use) navigation tools. These could provide functions similar to those readers are already familiar with in a paper environment (e.g. bookmarks, margin notes, paper clips) along with new functions which become necessary in the less tangible electronic environment.

The type of functionality that requires to be supported by such a suite of tools can be based on the notion that a reader traces a linear path (in time) through the material (chapters 4, 5 and 6 of (Kibby et al. 1991)):

Looking forward What subject areas are available in the current information collection, how much information is available in each, and how much information is likely to be relevant?

Moving forward Where can readers go, and how they can get there?

Current position Where is the current display located in the information space?

Moving back How can readers retrace their steps, either because they have reached a "dead-end", or because they want to revisit a screen?

Looking back Which material, and how much, has already been covered? 
Some form of graphical overview diagram would be able to convey much of this information, by representing the information space in some way and to show whether they have been visited or not, for example by shading-in different areas; possible future routes (perhaps derived from a query) could also be shown. The reader's current position can be given through the use of sufficient context information, or also by annotating an overview diagram. Other possible tools are bookmark and annotation lists.

An example of a suite of tools built in a working system can be seen in the NCSA Mosaic (Hughes 1994) program which has been created to allow the reader to browse through information distributed throughout the (internationally networked) world. It thus has to cope with handling a very large information space. There are specific navigation tools for going back and forth through already-visited nodes, and a separate, persistent list of bookmarks (called the Hotlist). For showing where the reader has already been (independent of the particular path taken) link markers are shaded differently.

The experiences gained from using a different, prototyped, suite of tools is given in chapter 10 of (Kibby et al. 1991). This suite of tools consisted of a history list, a means of marking the current screen in order to come back to it later ("bookmark"), the ability to view the list of marked screens (and go to a selected screen), and an indication in the history list of which screens were marked. When a screen was marked, for inclusion in the bookmark list, a symbol was also drawn on the screen itself. The conclusions from this work are that such tools are needed, but that in this particular study the readers did not spend sufficient time with the material, so they rarely needed to find their way back to previously studied sections. A probable explanation for this is that the tasks they were given were easily carried out with only the standard hyperlinks and no need was found to use the tools which had been supplied. This agrees with one of the conclusions in (Wright 1991), which states that users were grateful for the introduction of new tools only when they were given a problem that their current tool set was too weak to offer assistance.

\section{Improvements to authoring paradigms}

When designing a hypermedia presentation for the reader the author has to supply a good structure, a clear and aesthetically pleasing presentation and an adequate means of moving around and annotating the information. Given all these differing requirements, how can we make life easier for the author? We discuss briefly the requirements for authoring hypermedia after which we describe our hypermedia authoring system CMIFed.

\subsection{Authoring environment requirements}

Early hypermedia authoring systems did not support easy restructuring of the hypertext information - once the information structure had been created it was 
difficult to reorganise it. For example, the design of the tutorial in (Hardman 1988) was changed a number of times because the author was exploring the possibilities of the system she was using - Guide (OWL 1986). This meant that the information which had already created had to be laboriously cut and pasted into a new structure. Because of these inflexible authoring systems authors were constrained to get the hypertext design right the first time.

Another constraint imposed by most hypermedia authoring systems is that there is no separation of structure from layout - by placing an item on the screen the author specifies both its position in the presentation's structure and its layout attributes. The authoring process is simplified considerably if the layout of the items is under separate control. This is analogous to most word processors which allow the definition of different paragraph styles associated with different structure levels in the text.

A third feature which is often lacking in authoring systems is the ability to display the final presentation on a number of different platforms. This is a highly desirable feature - especially after the considerable amount of work the author has put into the creation of the presentation. This requires a platform independent representation of the presentation and the ability to re-create the author's intended layout styles for any end-user platform.

In summary, when creating a hypermedia presentation, including multiple, dynamic media such as sound and video, authors need to be able to combine items in a presentation, and to link from one presentation to another. Authors require some way of grouping nodes together, specifying timing constraints among the media items, creating links to a complete presentation and specifying screen layout.

\subsection{A Hypermedia Editor: CMIFed}

The CMIFed (CWI Multimedia Interchange Format editor) hypermedia authoring system (Van Rossum et al. 1993) was developed to satisfy the above requirements. It gives an author the ability to create explicit structure and timing relationships in a complex hypermedia document, and allows the editing of these in a straightforward way. The system uses a structured authoring approach (Hardman et al. 1993) where the author can create structure which can be filled in later with media items, or media items can be created and then later collected into one or more structures (although this flexibility does not preclude the need for a good design).

It is based on a platform-independent model of hypermedia (Hardman et al. 1994). This can be compared with the Dexter model (Halasz \& Schwartz 1994) which provides a similar level of description for hypertext, but does not include dynamic media, or the notion of combining nodes into presentation layouts (though does not exclude the latter). 
The CMIFed authoring environment separates out different tasks of the authoring process and presents the corresponding information in three separate, but connected, views. The hierarchy view allows the author to define the structural relations between the media items making up the presentation. This structure is used to derive basic timing information which is displayed in the channel view. The channel view shows the resource usage of the media items composing the presentation, and allows precise synchronization relations to be specified. The player is used to preview the presentation.

In order to describe the authoring environment of CMIFed we discuss it in terms of the following example. Figure 1 shows a "typical" multimedia presentation-a clip from a walking tour of Amsterdam. The presentation is formed from a collection of media items displayed on the screen, in this case an image and some text items. A spoken commentary, in either Dutch or English, is also given, and the subtitles change in time with the spoken words. The boxed text items (link markers) are linked to other parts of the multimedia presentation.

\section{Hierarchy View}

The hierarchy view, figure 2, is the primary authoring view, allowing the author to create multimedia presentations using a top-down or bottom-up approach. The hierarchically structured nodes of the presentation are represented as nested boxes, where children of a node are played either sequentially or in parallel.

Authoring is carried out by creating parallel and sequential structures (composite nodes) and assigning media items as the leaf nodes of this structure. Media items assigned at higher levels of the structure are displayed for the duration of the structure in which they are defined, e.g. the contents... and begin... items in figures 1,2 and 3.

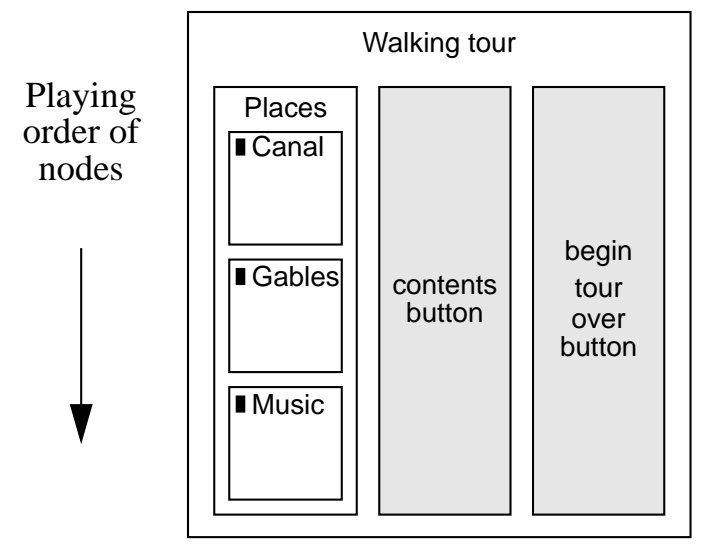

Figure 2. Hierarchy view for the Walking tour sequence.

Places, contents... and begin... are played in parallel. Canal, Gables and Music are played one after the other. A small dark box indicates hidden sub-structure. 


\section{Channel view}

CMIFed uses channels, logical output devices, upon which visual items can be displayed and audible items played. The channel view, figure 3 , shows the logical resource usage of a presentation, including timing relations derived from the structure defined in the hierarchy view. More complex timing constraints can be specified using synchronization arcs (the arrows in the figure). The media items making up the presentation are shown in the channel view with their precise durations and timing relationships.

A channel enables the author to define high-level presentation characteristics for each media type, so that presentations can be composed without having to specify details for each item: for example, a text channel defines a rectangular area on the screen and a font. Also, if the style of, for example, a heading is to be changed throughout the presentation then the change need be made only once for the relevant channel.

\section{Player}

The player allows the author to play a selection from the hierarchy or channel view, without having to go through a complete sequence. The author or end user can turn channels on and off, for example allowing the selection of alternative languages, e.g. UK and NL in figure 3.

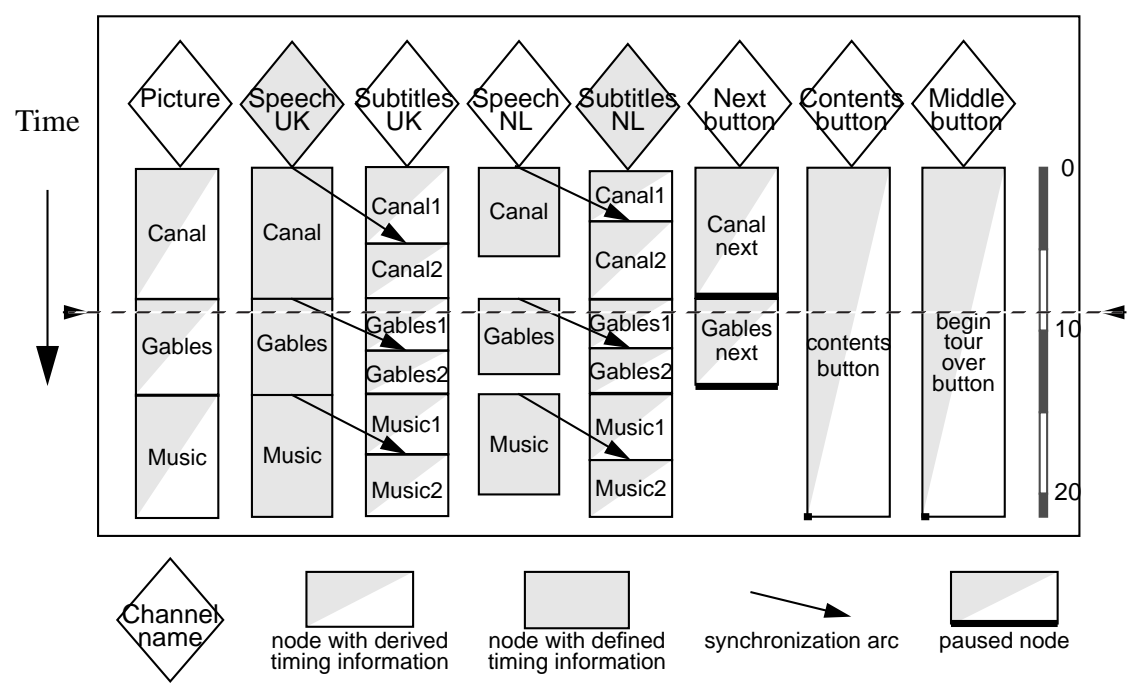

Figure 3. Channel View for the Walking tour sequence.

The diamonds at the top of the figure show the channel names (inactive channels are shaded). The data nodes assigned to the channels are represented as boxes beneath the diamonds. The height of a box represents its duration. A fully-shaded box has its duration explicitly defined, either through its data type, for sound and video, or through the author assigning a specific duration. A box with a shaded triangle has inherited its duration from its parent in the presentation's structure. 


\section{Future of Hypermedia Authoring}

From the work presented here we can analyse what is expected of a well-designed hypermedia presentation, we can separate out the different tasks required of the author, and we can produce an authoring system support these tasks explicitly. The author, however, is still required to do all the authoring work. A following step would be to allow the author to specify high-level requirements for the information and layout, and have the system generate the details of the presentation from this. The ultimate goal for high-volume applications, such as the daily news, would be that the end user becomes the author — defining their information requirements and having the system present the information with no further human intervention. (Haake et al. 1994) have already investigated this approach and related work is reported in this volume (Kamps \& Reichenberger 1994).

With automatic methods we cannot hope to replicate human creativity, but for large volume, lower quality applications we can aspire to reduce the tedium of the hypermedia author's task.

\section{References}

Edwards, D. M. \& Hardman, L. (1989). 'Lost in Hyperspace': Cognitive Mapping and Navigation in a Hypertext Environment. In R. McAleese (Ed.) Hypertext: theory into practice, pp. 105-125. Oxford: Intellect, 1989.

Evenson, S. \& Rheinfrank, J. (1989). Towards a Design Language for Representing Hypermedia Cues. In Proceedings of the 2nd ACM Conference on Hypertext (Hypertext'89), pp. 83-92, Pittsburg, PA, Nov. 5-8, 1989.

Garzotto, F., Mainetti, L. \& Paolini, P. (1994). Hypermedia Application Design: A Structured Approach. This volume.

Haake, A., Hüser, C. \& Reichenberger, K. (1994). The Individualized Electronic Newspaper: An Example of an Active Publication. To appear in Special Issue of Electronic Publishing: Origination, Dissemination and Design on Active Documents.

Halasz, F. \& Schwartz, M. (1994). The Dexter Hypertext Reference Model. Communications of the ACM, 37(2): 30-39.

Hannemann, J. \& Thüring, M. (1994). What Matters in Developing User Interfaces for Hyperdocument Presentation? This volume.

Hardman, L. (1988). Hypertext Tips: Experiences in Developing a Hypertext Tutorial. In Jones, D. M. \& Winder, R. (Eds.) People and Computers IV, pp. 437-451. Cambridge: Cambridge University Press (1988).

Hardman, L. (1989). Evaluating the usability of the Glasgow Online hypertext. Hypermedia 1(1): 34-63. 
Hardman, L. \& Sharratt, B. (1989). User-Centred Hypertext Design: the application of HCI design principles and guidelines. In R. McAleese and C. Green (Eds.) Hypertext State of the Art, pp. 252 - 259. Oxford: Intellect 1990.

Hardman, L., Van Rossum, G. \& Bulterman, D. C. A. (1993). Structured Multimedia Authoring. In Proceedings of the 1st ACM Conference on Multimedia (Multimedia '93), pp. 283-289, Anaheim, CA, Aug 1-6, 1993.

Hardman, L., Bulterman, D. C. A. \& Van Rossum, G. (1994). The Amsterdam Hypermedia Model: Adding Time and Context to the Dexter Model. Communications of the ACM, 37(2): 50-63.

Hughes K. (1994). Distributed Systems. SIGLink Newsletter, 3(1): 4-8.

Kahn, P. \& Lenk, K. (1993). Typography for the Computer Screen: Applying the Lessons of Print to Electronic Documents. Seybold Report on Desktop Publishing: 7(11): 3-16.

Kahn, P. (1994). Three Fundamental Elements of Visual Rhetoric in Hypertext. This volume.

Kamps, T. \& Reichenberger, K. (1994). A dialogue approach to graphical information access. This volume.

Kibby, M.R., Hardman, L., Tanner, G., Mayes, J.T., Knussen, C. \& Grant S. (1991). Final report on user interfaces for hypermedia. DELTA Project P7061 (D1014), The SAFE Project, HYP/21, March 1991. (Available from author.)

OWL (1986). Guide Users Manual. OWL International Inc., Bellevue, Washington, USA, 1986.

Nielsen, J. (1989). The Matters that Really Matter for Hypertext Usability. In Proceedings of the 2nd ACM Conference on Hypertext (Hypertext'89), pp. 239-248, Pittsburg, PA, Nov. 5-8, 1989.

Van Rossum, G., Jansen, J., Mullender, K. S. \& Bulterman, D. C. A. (1993). CMIFed: A Presentation Environment for Portable Hypermedia Documents. In Proceedings of the 1st ACM Conference on Multimedia (Multimedia '93), pp. 183-188, Anaheim, CA, Aug 1-6, 1993.

Wright, P. (1991). Cognitive Overheads and Prostheses: Some Issues in Evaluating Hypertexts. In Proceedings of the 3rd ACM Conference on Hypertext (Hypertext '91), pp. 1-12, San Antonio, TX, Dec. 15-18, 1991. 